\title{
RHOA mutation in diffuse-type gastric cancer: a comparative clinicopathology analysis of 87 cases
}

\author{
Tetsuo Ushiku' ${ }^{1}$ Shumpei Ishikawa ${ }^{2} \cdot$ Miwako Kakiuchi $^{3,4} \cdot$ Atsushi Tanaka $^{1}$ • \\ Hiroto Katoh $^{2} \cdot$ Hiroyuki Aburatani $^{3} \cdot$ Gregory Y. Lauwers $^{5} \cdot$ Masashi Fukayama $^{1}$
}

Received: 14 October 2014/ Accepted: 13 March 2015/Published online: 1 April 2015

(c) The Author(s) 2015. This article is published with open access at Springerlink.com

\begin{abstract}
Background Recent studies have discovered recurrent RHOA mutations in diffuse-type gastric cancers. These reports show mutant RhoA is an important cancer driver and is a potential therapeutic target. This study aims to investigate the clinicopathological features of diffuse-type gastric cancers with RHOA mutation.

Methods We performed a thorough review of 87 diffusetype gastric cancers, including 22 RHOA-mutated and 65 RHOA wild-type gastric cancers.

Results Most advanced tumors with RHOA mutation appeared as Borrmann type 3 lesions (81\%) developing in the middle $(50 \%)$ or distal $(32 \%)$ third of the stomach. Histologically, although all of the tumors were predominantly or exclusively composed of poorly cohesive carcinoma, limited tubular differentiation was also observed in $73 \%$ of the RHOA-mutated tumors. Notably, RHOA-mutated tumors more frequently showed a permeative growth pattern at the edge of the mucosal area (59\%)
\end{abstract}

Shumpei Ishikawa

sish.gpat@mri.tmd.ac.jp

1 Department of Pathology, Graduate School of Medicine, The University of Tokyo, Tokyo, Japan

2 Department of Genomic Pathology, Medical Research Institute, Tokyo Medical and Dental University, 1-5-45 Yushima, Bunkyo-ku, Tokyo 113-8510, Japan

3 Genome Science Division, Research Center for Advanced Science and Technology, The University of Tokyo, Tokyo, Japan

4 Department of Gastroenterology, Graduate School of Medicine, The University of Tokyo, Tokyo, Japan

5 Department of Pathology, Massachusetts General Hospital Boston, Boston, MA, USA compared with RHOA wild-type tumors (29\%, $P=0.0202$ ). Additionally, the size ratios of the deeply invasive components to the mucosal components were significantly lower in RHOA-mutated tumors [less than 1.45 (median) in $68 \%$ of cases] than in $R H O A$ wild-type tumors (less than 1.45 in $42 \%$ of cases, $P=0.0482$ ). RHOA mutation did not significantly impact survival in this study.

Conclusions These observations suggest that RHOA mutation may be associated with the growth patterns of diffuse-type gastric cancer but have a limited prognostic impact in isolation. Further studies, including analyses of the other alterations involving the RhoA pathways, such as CLDN18-ARHGAP fusion, as well as functional studies of mutant RhoA, are necessary to clarify the significance of alterations in the RhoA-signaling pathway in diffuse-type gastric cancers.

Keywords RHOA - Mutation - Gastric cancer - Diffuse type

\section{Introduction}

Gastric cancer remains the third leading cause of cancer death worldwide. Despite improvements in the treatment of gastric cancer, patients with advanced or metastatic disease have a poor prognosis, with 5-year survival rates of approximately $5-15 \%$ for stage IV disease $[1,2]$. The combination of chemotherapy and treatment with trastuzumab, which is an antibody against human epidermal growth factor receptor 2 (HER2), is thus far the only proven targeted therapy that is indicated for patients with HER2-positive gastric cancer [3]. Unfortunately, most patients with diffuse-type gastric cancer (according to the 
Lauren classification [4]) may not receive trastuzumab therapy because the HER2-positivity rates are only 2-7\% in this histological type [5-8]. Therefore, identification of a potential therapeutic target for these patients with aggressive diffuse-type gastric cancer would be particularly valuable.

Several recent studies involving the use of whole-exome or whole-genome sequencing have reported recurrent nonsynonymous mutations of $R H O A$ in a subset of gastric cancers [9-11]. RHOA, which encodes the small GTPase $\mathrm{RhoA}$, is a master regulator of actin-myosin-dependent cell contractility and cellular motility [12, 13]. Although until recently $R H O A$ has never been reported to be mutated in human cancers, its overexpression and association with tumor progression have been reported in various cancers [14-19]. Notably, RHOA mutation has been detected almost exclusively in diffuse-type gastric cancers, accounting for 14-25\% of the cases of this type, whereas it is absent in intestinal-type cancers [9-11]. The functional assays in our report using small interfering RNA knockdowns and rescue experiments have demonstrated the growth-promoting effects of mutant RhoA [9]. From these observations, mutant RhoA is likely to function in a gainof-function manner and play a key role in the carcinogenesis of diffuse-type gastric cancer. Importantly, RhoA could be a potential druggable oncogenic protein because it has various targetable domains, such as binding pockets for GTP and structural regions for protein-protein interactions with effectors, RhoGAPs and RhoGEFs. However, the previous studies have lacked thorough histological descriptions, and the clinicopathological significance of RHOA mutation in diffuse-type gastric cancer is largely unknown.

In this study, we performed a retrospective analysis of 87 cases of diffuse-type gastric cancer, including 22 RHOA-mutant and 65 RHOA wild-type cases, to better clarify the clinicopathological features of $R H O A$-mutated diffuse-type gastric cancer. In light of the previous reports that RhoA is associated with tumor cell motility and invasion in various types of cancers [18, 20, 21], we performed a histological review with a particular focus on the association between the RHOA mutation and growth patterns.

\section{Materials and methods}

\section{Study group}

The study was approved by the Institutional Review Board of Tokyo University Hospital. The cohort included 87 patients with diffuse-type gastric cancer with an established mutation status of the RHOA gene, which had been determined by whole-exome sequencing $(n=30)$ or targeted deep sequencing $(n=57)$ in our previous study [9]. Twenty-two of the 87 tumors $(25 \%)$ were RHOA mutated, and the remaining 65 tumors $(75 \%)$ were $R H O A$ wild type.

\section{Clinical data}

The demographic data, endoscopic findings, and clinical follow-up data were obtained by reviewing the medical records. Tumor staging was performed according to the tumor-node-metastasis classification system [22]. The macroscopic tumor type was classified according to the criteria of the World Health Organization classification for early gastric cancer and the Borrmann classification for advanced gastric cancer [22].

\section{Histological evaluation}

All the assessments were performed on the basis of the examination of the histological sections of the primary tumor by a gastrointestinal pathologist (T.U.) without knowledge of the mutation status and outcome of the patients. The number of hematoxylin and eosin stained sections per tumor ranged from 3 to 36 (mean 12.6; median 11). The histological features were recorded: histological type, stromal features, growth pattern, tumor size, tumor stage, lymphovascular invasion, perineural invasion, and nodal metastasis. The histological type was determined according to the criteria of the World Health Organization classification: tubular, papillary, mucinous, and poorly cohesive carcinomas [22]. Although all the tumors that were included in this study consisted predominantly or exclusively of poorly cohesive carcinoma because this study included only diffuse-type gastric cancers, other histological types were also partially recognized and were recorded. The degree of lymphovascular invasion was scored as follows: none, minimal, moderate, and marked. Stromal features that were evaluated in the study included desmoplasia, myxoid change, and inflammation. The growth pattern at the advancing edge of the deeply invasive area was classified into two types: expanding and infiltrative types [23]. In addition, the growth pattern of the intramucosal area, which is usually different from that of the deeply invasive area, was evaluated separately; the pattern was identified as "permeative" when the neoplastic cells infiltrated between the normal pits or glands with no recognizable margin to the growth, and "expansile" when the growing margin was sharply delineated and the tumor had a well-defined margin at the advancing edge. In addition, tumor size in each case was measured separately in the mucosal and deeply invasive components in the following procedure. First, we took gross photographs of the mucosal surface and cut surfaces of the tumor, and then each section 
for histological examination was marked on a printed photograph. In advanced tumors, we took sections in 5-mm slices including the greatest dimension and the deepest penetration of tumor. Additional sections perpendicular to the section of the greatest dimension were taken to figure out the spread of the tumor, and were submitted for histological evaluation. In early cancers, entire tumor was cut in 3-4-mm slices parallel to the lesser curvature, and all the sections were submitted for histological evaluation. After histological evaluation, the cancerous area was marked on the gross photographs of the cut surface as well as the mucosal surface to demonstrate the spread of the tumor accurately. Finally, we measured the size of the mucosal and deeper invasive components separately, and the size ratios of the deeper invasive components to the intramucosal components were calculated. In tumors with central ulceration, the intramucosal component remained at the ulcer edge at least in a small amount, in which case the size of the intramucosal components was defined as the total size of the intramucosal tumor at the ulcer edge and ulceration.

\section{Immunohistochemical studies}

Formalin-fixed paraffin-embedded tissue blocks were available for all 87 cases. To determine the tumor immunophenotype, immunohistochemical staining was performed using antibodies (clone, dilution, manufacturer) for mucin 2 (Muc2) (CLH2, 1:500, Novocastra Laboratories, Newcastle, UK), CD10 (56C6, 1:100, Novocastra Laboratories), mucin 5AC (Muc5AC) (CLH5, 1:500, Novocastra Laboratories), and mucin 6 (Muc6) (CCP58, 1:500, Novocastra Laboratories). Immunohistochemical staining was performed using a Ventana Benchmark XT autostainer (Ventana Medical Systems, Tucson, AZ) with the labeled streptavidin-biotin peroxidase method, and the signals were visualized with 3,3'-diaminobenzidine.

Cytoplasmic staining for mucin core proteins and apical membranous staining for CD10 were evaluated. The tumor was defined as positive for each marker when more than $10 \%$ of the neoplastic cells were stained, as reported previously [24]. On the basis of the immunohistochemistry, tumors were categorized into the gastric (Muc5 $\mathrm{AC}^{+}$and/or Muc6 $^{+}$; Muc2 $2^{-}$and $\mathrm{CD}_{10}^{-}$), intestinal Muc2 $^{+}$and/or $\mathrm{CD}_{10}^{+}$; Muc5 $\mathrm{AC}^{-}$and $\mathrm{Muc6}^{-}$), mixed $\left(\mathrm{Muc2}^{+}\right.$and/or $\mathrm{CD}_{10}{ }^{+} ; \mathrm{Muc} \mathrm{AC}^{+}$and/or Muc6 ${ }^{+}$), and null (all negative) types.

\section{Statistical analysis}

The clinicopathology data were compared by Fisher's exact test or the chi square test for categorical variables, and Student's $t$ test or the Mann-Whitney $U$ test for continuous variables. Survival curves were calculated by the KaplanMeier method and were compared using the log-rank test. Multivariate Cox proportional hazard models were used to identify the variables that were associated with diseasespecific and disease-free survivals. Differences were considered significant when the $P$ value from the two-tailed test was less than 0.05 . Statistical analyses were performed with Excel Statistics (SSRI, Tokyo, Japan).

\section{Results}

\section{Clinical characteristics of $\mathrm{RHOA}$-mutated diffuse- type gastric cancer}

The clinical features of the RHOA-mutant and RHOA wildtype tumors are summarized in Table 1 . The patients with RHOA-mutant tumors included 13 men and nine women, with a mean age of 65 years (range $40-84$ years). The anatomic distribution of the tumors was as follows: upper third, 4 (18\%); middle third, 11 (50\%); and lower third, 7 (32\%). Tumor sizes ranged from 2.2 to $15 \mathrm{~cm}$ (mean $6.4 \mathrm{~cm}$; median $6.0 \mathrm{~cm}$ ). Endoscopically, all the early cancers had the appearance of a superficial depressed lesion. Most of the advanced cancers were Borrmann type 3 lesions $(n=13,81 \%)$, and the remaining ones were Borrmann type 4 lesions $(n=3,19 \%)$. Six tumors with mutant RHOA (27\%) were early cancers (T1), whereas the other 16 tumors $(73 \%)$ were advanced cancers (T2-T4). Nodal metastases were noted in 16 cases $(73 \%)$. These features were not significantly different from those of RHOA wild-type tumors (Table 1).

\section{Histological features of RHOA-mutated diffuse-type gastric cancers}

The associations between the RHOA mutation and histological characteristics are summarized in Table 2. The histological type of the RHOA-mutated tumors was less frequently pure poorly cohesive carcinoma than for the RHOA wild-type tumors ( $27 \%$ vs $46 \%$ ), although this did not reach statistical significance $(P=0.1201)$ (Fig. 1a). Signet ring cell carcinoma, a major variant of poorly cohesive carcinoma, was identified in 18 of the 22 RHOA-mutated tumors $(82 \%)$ and in 52 of the 65 RHOA wild-type tumors $(80 \%)$ (Fig. 1b). RHOA-mutated tumors showed focal tubular differentiation in 16 of the 22 cases $(73 \%)$, including three (14\%) with mucinous differentiation (Fig. 1c, d). RHOA wild-type tumors also had focal tubular and/or mucinous differentiation in approximately half of the cases. Tubular differentiation within the mucosal area was more frequently observed in $R H O A$-mutated tumors (16 of $22,73 \%$ ) than in RHOA wild-type tumors (28 of $65,43 \%$, 
Table 1 Clinicopathological features of RHOA-mutant and RHOA wild-type diffuse-type gastric cancer

\begin{tabular}{|c|c|c|c|}
\hline Characteristics & RHOA mutated $(n=22)$ & $R H O A$ wild type $(n=65)$ & $P$ \\
\hline \multicolumn{4}{|l|}{ Sex } \\
\hline Male & $13(59 \%)$ & $38(58 \%)$ & \multirow[t]{2}{*}{1} \\
\hline Female & $9(41 \%)$ & $27(42 \%)$ & \\
\hline Mean age and range (years) & $65(40-84)$ & $63(30-85)$ & 0.5462 \\
\hline \multicolumn{4}{|l|}{ Locus } \\
\hline Proximal third & $4(18 \%)$ & $16(25 \%)$ & \multirow[t]{3}{*}{0.7894} \\
\hline Middle third & $11(50 \%)$ & $28(43 \%)$ & \\
\hline Distal third & $7(32 \%)$ & $21(32 \%)$ & \\
\hline Mean tumor size $\pm \mathrm{SD}(\mathrm{mm})$ & $6.4 \pm 3.3$ & $9.1 \pm 8.1$ & 0.1352 \\
\hline \multicolumn{4}{|l|}{ Macroscopic type } \\
\hline \multicolumn{4}{|l|}{ Early cancer } \\
\hline Superficially depressed type & $6(100 \%)$ & $12(100 \%)$ & 1 \\
\hline \multicolumn{4}{|l|}{ Advanced cancer } \\
\hline Borrmann type 2 & 0 & $2(4 \%)$ & \multirow[t]{3}{*}{0.5115} \\
\hline Borrmann type 3 & $13(81 \%)$ & $36(68 \%)$ & \\
\hline Borrmann type 4 & $3(19 \%)$ & $15(28 \%)$ & \\
\hline \multicolumn{4}{|l|}{ T stage } \\
\hline T1a, T1b & $6(27 \%)$ & $12(18 \%)$ & \multirow[t]{4}{*}{0.4132} \\
\hline $\mathrm{T} 2$ & $2(9 \%)$ & $2(3 \%)$ & \\
\hline $\mathrm{T} 3$ & $3(14 \%)$ & $16(25 \%)$ & \\
\hline $\mathrm{T} 4 \mathrm{a}, \mathrm{T} 4 \mathrm{~b}$ & $11(50 \%)$ & $35(54 \%)$ & \\
\hline \multicolumn{4}{|l|}{$\mathrm{N}$ stage } \\
\hline No & $7(32 \%)$ & $27(42 \%)$ & \multirow[t]{4}{*}{0.4601} \\
\hline N1 & $3(14 \%)$ & $6(9 \%)$ & \\
\hline $\mathrm{N} 2$ & $4(18 \%)$ & $10(15 \%)$ & \\
\hline N3 & $8(36 \%)$ & $22(34 \%)$ & \\
\hline \multicolumn{4}{|l|}{ Peritoneal dissemination } \\
\hline Present & $5(23 \%)$ & $15(23 \%)$ & \multirow[t]{2}{*}{1} \\
\hline Absent & $17(77 \%)$ & $50(77 \%)$ & \\
\hline \multicolumn{4}{|l|}{ M stage (distant metastasis) } \\
\hline Present & $1(5 \%)$ & $5(8 \%)$ & \multirow[t]{2}{*}{1} \\
\hline Absent & $21(95 \%)$ & $60(92 \%)$ & \\
\hline \multicolumn{4}{|l|}{ Stage } \\
\hline I & $6(27 \%)$ & $11(17 \%)$ & \multirow[t]{4}{*}{0.7457} \\
\hline II & $5(23 \%)$ & $19(29 \%)$ & \\
\hline III & $5(23 \%)$ & $17(26 \%)$ & \\
\hline IV & $6(27 \%)$ & $18(28 \%)$ & \\
\hline
\end{tabular}

$S D$ standard deviation

$P=0.0254$ ), whereas tubular differentiation in the submucosa or deeper area was noted at similar frequencies in the two groups ( $53 \%$ vs $46 \%, P=0.7922$ ). There were no significant differences in the stromal features and the extent of lymphovascular invasion and perineural invasion between the two groups. Notably, four RHOA-mutated tumors $(18 \%)$ and eight RHOA wild-type tumors (12\%, $P=0.4893$ ) demonstrated carcinomatous lymphangiosis, which was characterized by prominent lymphatic involvement in the full thickness of the gastric wall with dilated lymphatics filled with neoplastic cells.

RHOA-mutated tumors demonstrated distinct morphological features in terms of the growth pattern. An intramucosal permeative growth pattern was more frequently observed in $R H O A$-mutated tumors than in RHOA wildtype tumors, with a significant difference (59\% vs $29 \%$, $P=0.0202$ ) (Fig. 2). The growth patterns in the deeply invasive area did not differ between the two groups, both of which usually demonstrated an infiltrative growth pattern. However, the size ratios of the submucosal or deeper invasive area to the intramucosal area were significantly lower in cases with RHOA mutation [less than 1.45 (median) in $68 \%$ ] than in those without RHOA mutation (less than 1.45 in $42 \%, P=0.0482$ ). There was a significant difference in the ratios of deeply invasive to intramucosal size between the two groups when compared by the MannWhitney $U$ test $(P=0.0308)$. Our cohort included five cases of linitis plastica type cancer, which is characterized 
Table 2 Histological features of diffuse-type gastric cancers with or without $R H O A$ mutation

\begin{tabular}{|c|c|c|c|}
\hline Findings & RHOA mutated $(n=22)$ & RHOA wild type $(n=65)$ & $P$ \\
\hline \multicolumn{4}{|l|}{ Histological type } \\
\hline Pure poorly cohesive carcinoma & $6(27 \%)$ & $30(46 \%)$ & 0.1201 \\
\hline Poorly cohesive plus other types & $16(73 \%)$ & $35(54 \%)$ & \\
\hline Presence of tubular component & $16(73 \%)$ & $33(51 \%)$ & 0.0862 \\
\hline Intramucosal area & $16(73 \%)$ & $28(43 \%)$ & 0.0254 \\
\hline Submucosal or deeper area & $10(53 \%)$ & $27(46 \%)$ & 0.7922 \\
\hline Presence of mucinous component & $3(14 \%)$ & $9(14 \%)$ & 1.0000 \\
\hline Stromal features & & & 0.3575 \\
\hline Desmoplastic & $18(82 \%)$ & $58(89 \%)$ & \\
\hline Inflammatory & $9(41 \%)$ & $13(20 \%)$ & \\
\hline Myxoid & $5(23 \%)$ & $14(22 \%)$ & \\
\hline Normal & $4(18 \%)$ & $6(9 \%)$ & \\
\hline \multicolumn{4}{|l|}{ Lymphatic invasion } \\
\hline Negative or minimal & $12(55 \%)$ & $39(60 \%)$ & 0.8028 \\
\hline Moderate or marked & $10(45 \%)$ & $26(40 \%)$ & \\
\hline \multicolumn{4}{|l|}{ Vascular invasion } \\
\hline Negative or minimal & $13(59 \%)$ & $36(55 \%)$ & 0.8082 \\
\hline Moderate or marked & $9(41 \%)$ & $29(45 \%)$ & \\
\hline Carcinomatous lymphangiosis & $4(18 \%)$ & $8(12 \%)$ & 0.4893 \\
\hline Perineural invasion & $7(32 \%)$ & $31(48 \%)$ & 0.2226 \\
\hline \multicolumn{4}{|l|}{ Growth pattern } \\
\hline \multicolumn{4}{|l|}{ Intramucosal area } \\
\hline Permeative & $13(59 \%)$ & $19(29 \%)$ & 0.0202 \\
\hline Expansile & $9(41 \%)$ & $46(71 \%)$ & \\
\hline \multicolumn{4}{|l|}{ Submucosa or deeper area } \\
\hline Infiltrative & $19(100 \%)$ & $58(98 \%)$ & 1.0000 \\
\hline Expanding & 0 & $1(2 \%)$ & \\
\hline \multicolumn{4}{|c|}{ Ratio of deeply invasive to intramucosal size } \\
\hline$\geq 1.45^{\mathrm{a}}$ & $6(32 \%)$ & $34(58 \%)$ & 0.0482 \\
\hline$<1.45$ & $13(68 \%)$ & $25(42 \%)$ & \\
\hline
\end{tabular}

${ }^{\text {a }}$ Median of the ratios of deeply invasive to intramucosal size as a leather bottle-like Borrmann type 4 tumor with relatively small intramucosal components in proportion to the extensive gastric wall involvement, and all of these cases were of the RHOA wild type (Fig. 3).

\section{Correlation of RHOA mutation with immunophenotype}

On the basis of immunostains for gastric phenotypic markers (Muc5AC and Muc6) and intestinal phenotypic markers (Muc2 and CD10), the 22 RHOA-mutated tumors were classified as gastric type $(n=10,45 \%)$, mixed type ( $n=8,36 \%)$, intestinal type $(n=1,5 \%)$, and null type $(n=3,14 \%)$. The 65 RHOA wild-type tumors were classified as gastric type $(n=31,48 \%)$, mixed type $(n=22,34 \%)$, intestinal type $(n=7,11 \%)$, and null type $(n=5,7 \%)$. There was no significant difference in the frequencies of each phenotype between the two groups.

\section{Patient outcome and prognostic factors}

Follow-up information for 1-126.7 months (mean 46.8 months) was available for all of the cases. All the patients with stage I disease $(n=17)$ were alive without disease at the last contact (range 17-126.7 months; mean 78.1 months), except for one patient, who died of another disease. Patients with stage II-IV disease $(n=70)$ had either died of the disease $(n=33)$, were alive without the disease $(n=28)$, were alive with the disease $(n=6)$, or had died of another disease $(n=3)$. Noticeably, a single patient with $R H O A$-mutated early cancer (pT1b) developed pulmonary hypertension due to pulmonary tumor thrombotic microangiopathy that was caused by gastric cancer, which was revealed by video-assisted thoracic surgery and subsequent autopsy.

A survival analysis was performed on the patients with stage II-IV disease. In univariate analyses, the Kaplan- 
Fig. 1 Histology of $R H O A$ mutated gastric cancers. Poorly cohesive carcinoma is the predominant component (a), including signet-ring cells in many cases (b). Focal tubular differentiation is frequently recognized (c), and a mucinous component may also be present (d)
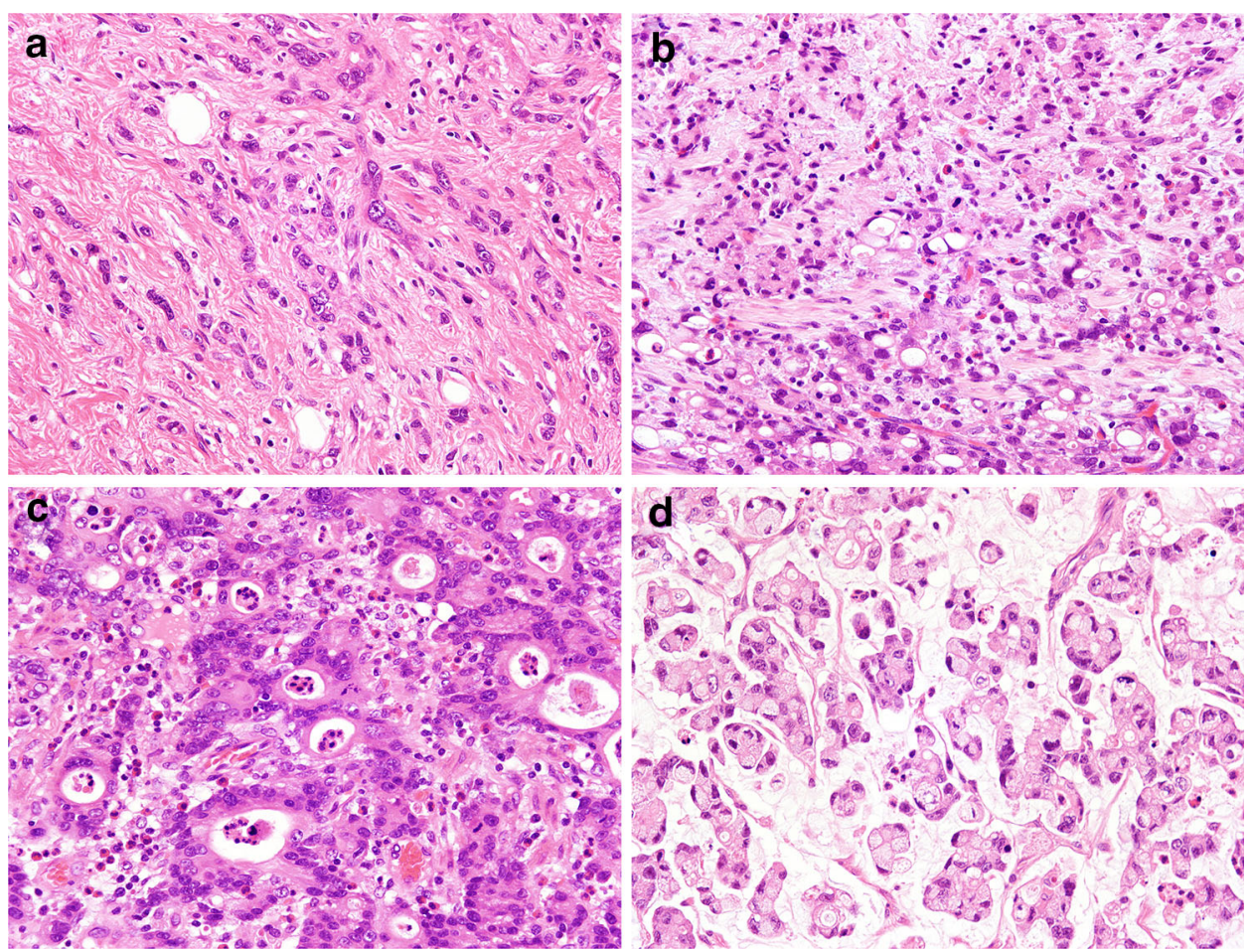

Meier survival curves demonstrated that RHOA mutation was not significantly associated with disease-specific survival ( $P=0.3507$ by the log-rank test) or disease-free survival $(P=0.9813)$ (Fig. 4). Stage IV disease (vs stage II-III disease), the presence of lymphatic invasion, and lymph node metastasis were associated with decreased disease-specific survival $(P<0.0001,0.0003$, and 0.0004 , respectively) and disease-free survival $(P=0.0029$, 0.0016 , and 0.0111 , respectively). Other features, including gender, tumor size, $\mathrm{T}$ stage, venous invasion, and perineural invasion, were not associated with differences in disease-specific survival or disease-free survival. In multivariate analyses, RHOA mutation was not a significant prognostic factor.

\section{Discussion}

Comprehensive genomic analyses have recently increased our understanding of gastric cancer [9-11]. The largest study to date, conducted by The Cancer Genome Atlas (TCGA) project, proposed a molecular classification that divided gastric cancer into four subtypes: tumors positive for Epstein-Barr virus, microsatellite-unstable tumors, genomically stable tumors, and tumors with chromosomal instability [10]. Genomically stable tumors, which nearly correspond to diffuse-type cancers in terms of histological features, were characterized by mutations of $R H O A$ or CLDN18-ARHGAP6/ARHGAP26 fusions in addition to the well-known mutations of $C D H 1$. Within the genomically stable subgroup, $30 \%$ of the cases had either RHOA or CLDN18-ARHGAP alterations. Furthermore, analyses of the gene expression status in the RhoA-signaling pathways suggested that these genomic alterations resulted in the activation of the RhoA-driven pathways [10]. These results obtaiend by the TCGA project are consistent with our previous study demonstrating the recurrent RHOA mutations exclusively in diffuse-type gastric cancer $(25 \%$ of the cases) [9]. Our small interfering RNA knockdown and rescue experiments showed growth-promoting effects of mutant RHOA, suggesting a gain-of-function role for RHOA mutations in progression of diffuse-type gastric cancers [9]. On the other hand, another report with a functional study suggested that mutant RHOA might cause defective RhoA signaling, which would promote escape from anoikis, an important early step in the carcinogenesis of diffuse-type gastric cancers [11]. The details of the functional consequences of $R H O A$ mutation in diffuse-type gastric cancer still remain largely unknown, and further research will be required to provide a more thorough understanding of the role of RHOA mutation in diffuse-type gastric cancer.

This study was the first to perform a thorough clinicopathology review of $R H O A$-mutated gastric cancers. From our observations, advanced RHOA-mutated tumors were characterized as Borrmann type 3 lesions $(81 \%)$ that developed in the middle third (50\%) or distal third (32\%) of the stomach. Histologically, tubular differentiation was 

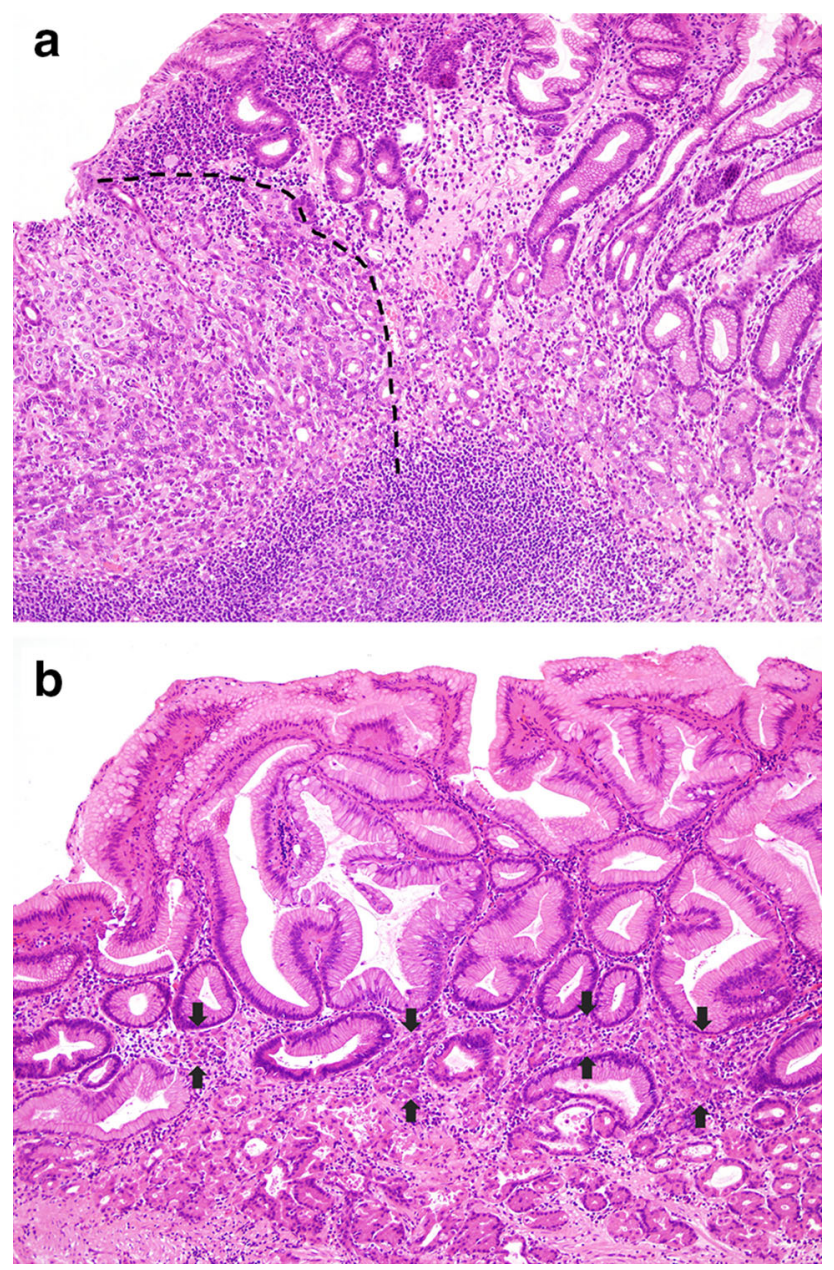

Fig. 2 Growth patterns at the edge of the intramucosal component. The expansile pattern demonstrates destructive invasion with a relatively well-defined margin (indicated by the dotted line) at the advancing edge (a). In the permeative pattern, neoplastic cells infiltrate between the normal pits or glands in the middle layer of the lamina propria, with no recognizable margin to the growth (b). Neoplastic cells are indicated by arrows

frequently observed $(73 \%)$ in addition to predominant poorly cohesive carcinoma. Notably, RHOA-mutated tumors more frequently showed permeative growth patterns at the edge of the mucosal area than did RHOA wild-type tumors, with a significant difference. In addition, the size ratio of the mucosal components to the deeply invasive components was significantly higher in tumors with $R H O A$ mutation than in those without RHOA mutation. Lnitis plastica type cancers, which are typically characterized as a leather bottle-like (Borrmann type 4) appearance, relatively small mucosal lesions in proportion to the extensive spread in the gastric wall, and histologically pure poorly cohesive carcinoma, were of the RHOA wild type in our cohort.

RHOA mutation may contribute to the intramucosal permeative growth pattern, potentially resulting in a relatively large mucosal component in proportion to the

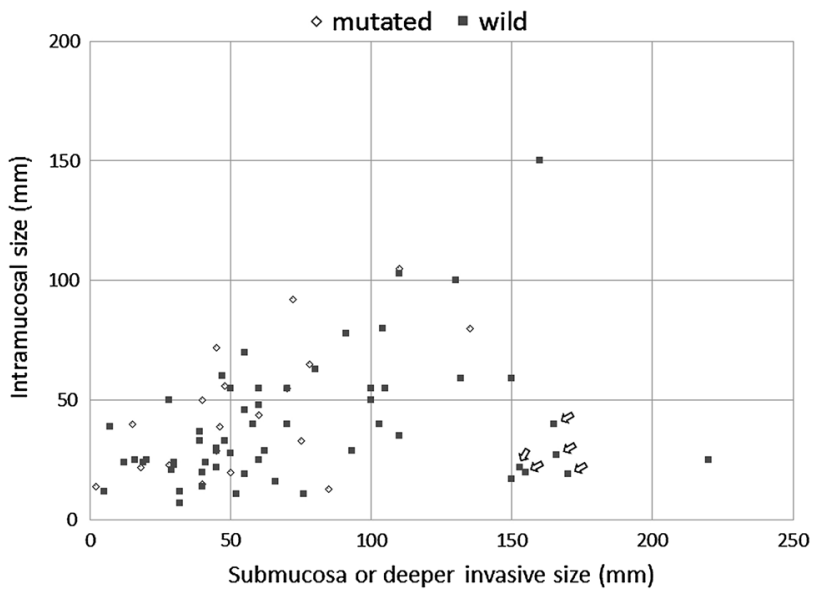

Fig. 3 Correlation between the sizes of the intramucosal components and those of the submucosal or deeper areas of each case. Arrows at the lower right indicate cases of linitis plastica type cancer $(n=5)$

deeply invasive area. First, RhoA is a critical regulator of actin-myosin-dependent cell contractility and cellular motility $[12,13,21]$. In particular, RhoA signaling drives amoeboid motility, which is characterized by protease-independent cellular movement, i.e., via propulsive squeezing through gaps of the extracellular matrix using an actomyosin-related contractile force [25, 26]. Second, RHOA mutation has been reported to be an early event in carcinogenesis, suggesting that tumor cells harbor the mutation at the early stage in the mucosa [9]. Therefore, it is possible that the intramucosal permeative growth pattern might reflect alterations in RhoA signaling. In addition, RhoA alterations could be associated with lymphovascular invasion, causing carcinomatous lymphangiosis or pulmonary tumor thrombotic microangiopathy in some extreme cases because RhoA is important in the transendothelial migration of neoplastic cells [27-29]. This hypothesis, however, remains speculative because there is a lack of data supporting an association between RHOA mutation and lymphovascular invasion in this study. Further functional studies are necessary to clarify the role of RHOA mutation in diffuse-type gastric cancers, particularly to develop therapeutic agents that target mutant RhoA.

RHOA mutation did not appear to have a significant impact on the survival in this study. The relatively small number of cases was an inherent limitation of our study. A larger sample size is necessary to verify the prognostic importance of the RHOA mutation. In addition, it is also important to include analyses of other genomic alterations that affect the RhoA-signaling pathway, such as CLDN18ARHGAP fusion, which has been predicted to alter RhoAdriven pathways as well as RHOA mutation [10]. Furthermore, TCGA data have suggested that there may be additional events within the genome-stable subgroup that result in RhoA-signaling activation because alterations in the 
Fig. 4 Kaplan-Meier survival plot according to $R H O A$ mutation status among patients with the stage II-IV disease. RHOA mutation was not significantly associated with disease-specific survival (a) or disease-free survival (b) of patients with diffuse-type gastric cancer
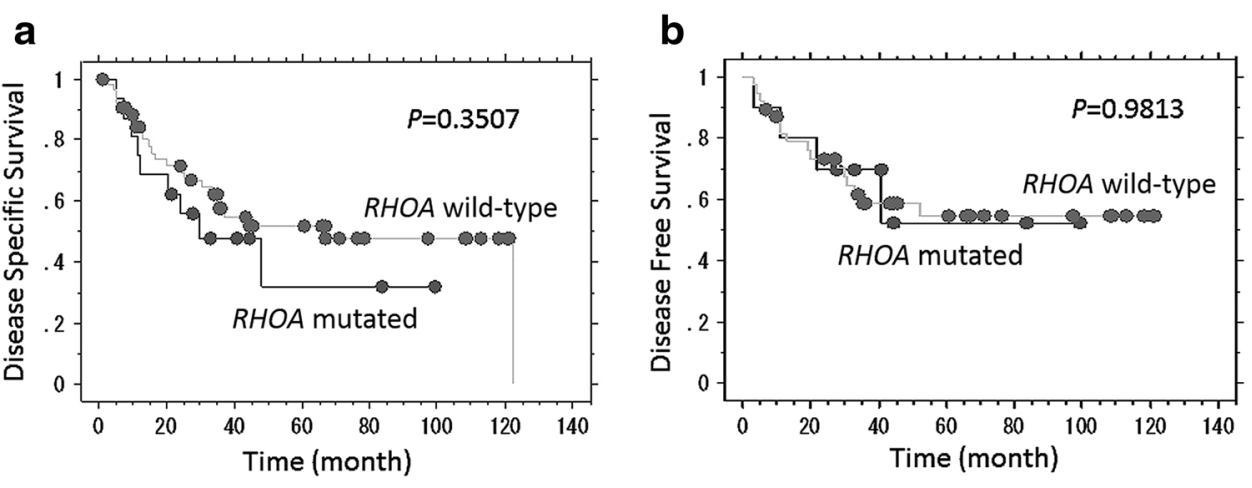

RhoA pathway are present in cases without $R H O A$ mutation or ARHGAP fusion as well [10]. Therefore, although RHOA mutation may not be a significant prognostic factor in isolation, analyses that include other genetic alterations involving RhoA pathways would better clarify the significance of the RhoA-signaling alterations.

In summary, advanced diffuse-type gastric cancers with RHOA mutation were characterized as Borrmann type 3 tumors with relatively large intramucosal components in proportion to deeply invasive components, frequent tubular differentiation in addition to predominant poorly cohesive carcinoma, and an intramucosal permeative growth pattern. Although RHOA mutation did not significantly impact the survival in the relatively small number of patients, further studies that include analyses of other alterations involving RhoA-signaling pathways, as well as a larger sample size of cases, are necessary to determine the significance of alterations in the RhoA-signaling pathway.

Acknowledgments This study was supported by the Project for Development of Innovative Research on Cancer Therapeutics (PDirect), Grants-in-Aid for Scientific Research in Innovative Areas to the "Systems Cancer Project" (grant 22134003) from the Ministry of Education, Culture, Sports, Science and Technology of Japan (S.I.), and by Grant-in-Aid for Scientific Research (A) (grant 26253021) from Japan Society for the Promotion of Science (M.F.).

Conflict of interest The authors have declared no conflicts of interest.

Open Access This article is distributed under the terms of the Creative Commons Attribution License which permits any use, distribution, and reproduction in any medium, provided the original author(s) and the source are credited.

\section{References}

1. Hundahl SA, Phillips JL, Menck HR. The National Cancer Data Base report on poor survival of US gastric carcinoma patients treated with gastrectomy: fifth edition American Joint Committee on Cancer staging, proximal disease, and the "different disease" hypothesis. Cancer. 2000;88:921-32.
2. Nashimoto A, Akazawa K, Isobe Y, Miyashiro I, Katai H, Kodera ZY, et al. Gastric cancer treated in 2002 in Japan: 2009 annual report of the JGCA nationwide registry. Gastric Cancer. 2013;16:1-27.

3. Bang YJ, Van Cutsem E, Feyereislova A, Chung HC, Shen L, Sawaki A, et al. Trastuzumab in combination with chemotherapy versus chemotherapy alone for treatment of HER2-positive advanced gastric or gastro-oesophageal junction cancer (ToGA): a phase 3, open-label, randomised controlled trial. Lancet. 2010;376:687-97.

4. Lauren $P$. The two histological main types of gastric carcinoma: diffuse and so-called intestinal-type carcinoma. An attempt at a histo-clinical classification. Acta Pathol Mcrobiol Scand. 1965;64:31-49.

5. Gravalos C, Jimeno A. HER2 in gastric cancer: a new prognostic factor and a novel therapeutic target. Ann Oncol. 2008;19:1523-9.

6. Hede K. Gastric cancer: trastuzumab trial results spur search for other targets. J Natl Cancer Inst. 2009;101:1306-7.

7. Tanner M, Hollmen M, Junttila TT, Kapanen AI, Tommola S, Soini Y, et al. Amplification of HER-2 in gastric carcinoma: association with topoisomerase II $\alpha$ gene amplification, intestinal type, poor prognosis and sensitivity to trastuzumab. Ann Oncol. $2005 ; 16: 273-8$.

8. Yan B, Yau EX, Bte Omar SS, Ong CW, Pang B, Yeoh KG, et al. A study of HER2 gene amplification and protein expression in gastric cancer. J Clin Pathol. 2010;63:839-42.

9. Kakiuchi M, Nishizawa T, Ueda H, Gotoh K, Tanaka A, Hayashi A, et al. Recurrent gain-of-function mutations of RHOA in diffuse-type gastric carcinoma. Nat Genet. 2014;46:583-7.

10. The Cancer Genome Atlas Research Network. Comprehensive molecular characterization of gastric adenocarcinoma. Nature. 2014;513:202-9.

11. Wang K, Yuen ST, Xu J, Lee SP, Yan HH, Shi ST, et al. Wholegenome sequencing and comprehensive molecular profiling identify new driver mutations in gastric cancer. Nat Genet. 2014;46:573-82.

12. Ridley AJ, Schwartz MA, Burridge K, Firtel RA, Ginsberg MH, Borisy $\mathrm{G}$, et al. Cell migration: integrating signals from front to back. Science. 2003;302:1704-9.

13. Thumkeo D, Watanabe $S$, Narumiya $S$. Physiological roles of Rho and Rho effectors in mammals. Eur J Cell Biol. 2013;92:303-15.

14. Faried A, Faried LS, Usman N, Kato H, Kuwano H. Clinical and prognostic significance of RhoA and RhoC gene expression in esophageal squamous cell carcinoma. Ann Surg Oncol. 2007;14:3593-601.

15. Horiuchi A, Imai T, Wang C, Ohira S, Feng Y, Nikaido T, et al. Up-regulation of small GTPases, RhoA and RhoC, is associated with tumor progression in ovarian carcinoma. Lab Investig. 2003;83:861-70. 
16. Kamai T, Tsujii T, Arai K, Takagi K, Asami H, Ito Y, et al. Significant association of Rho/ROCK pathway with invasion and metastasis of bladder cancer. Clin Cancer Res. 2003;9:2632-41.

17. Kamai T, Yamanishi T, Shirataki H, Takagi K, Asami H, Ito Y, et al. Overexpression of RhoA, Rac1, and Cdc42 GTPases is associated with progression in testicular cancer. Clin Cancer Res. 2004;10:4799-805.

18. Karlsson R, Pedersen ED, Wang Z, Brakebusch C. Rho GTPase function in tumorigenesis. Biochim Biophys Acta. 2009;1796:91-8.

19. Li XR, Ji F, Ouyang J, Wu W, Qian LY, Yang KY. Overexpression of RhoA is associated with poor prognosis in hepatocellular carcinoma. Eur J Surg Oncol. 2006;32:1130-4.

20. Matsuoka T, Yashiro M, Kato Y, Shinto O, Kashiwagi S, Hirakawa $\mathrm{K}$. RhoA/ROCK signaling mediates plasticity of scirrhous gastric carcinoma motility. Clin Exp Metastasis. 2011;28:627-36.

21. Ridley AJ. RhoA, RhoB and RhoC have different roles in cancer cell migration. J Microsc. 2013;251:242-9.

22. Lauwers GY, Carneiro F, Graham DY, Curado MP, Franceschi S, Montgomery E, et al. Gastric carcinoma. In: Bosman FT, Carneiro F, Hruban RH, Theise ND, editors. WHO classification of tumours of the digestive system. Lyon: IARC Press; 2010. p. $48-58$.
23. Ming SC. Gastric carcinoma. A pathobiological classification. Cancer. 1977;39:2475-85.

24. Tsukashita S, Kushima R, Bamba M, Sugihara H, Hattori T. MUC gene expression and histogenesis of adenocarcinoma of the stomach. Int J Cancer. 2001;94:166-70.

25. Wolf K, Mazo I, Leung H, Engelke K, von Andrian UH, Deryugina EI, et al. Compensation mechanism in tumor cell migration: mesenchymal-amoeboid transition after blocking of pericellular proteolysis. J Cell Biol. 2003;160:267-77.

26. Parri M, Chiarugi P. Rac and Rho GTPases in cancer cell motility control. Cell Commun Signal. 2010;8:23.

27. Worthylake RA, Lemoine S, Watson JM, Burridge K. RhoA is required for monocyte tail retraction during transendothelial migration. J Cell Biol. 2001;154:147-60.

28. Reymond N, Riou P, Ridley AJ. Rho GTPases and cancer cell transendothelial migration. Methods MolBiol. 2012;827:123-42.

29. Roh-Johnson M, Bravo-Cordero JJ, Patsialou A, Sharma VP, Guo $\mathrm{P}$, Liu $\mathrm{H}$, et al. Macrophage contact induces RhoA GTPase signaling to trigger tumor cell intravasation. Oncogene. 2014;33:4203-12. 